\title{
Suggestions Regarding the Effective Temperatures and Bolometric Corrections for B-type Stars
}

\author{
ANNE B. UNDERHILL \\ Sonnenborgh Observatory, Utrecht, The Netherlands
}

\section{Summary}

Bolometric corrections and effective temperatures are presented for fourteen model atmospheres which are believed to represent main-sequence B-type stars. The models are in radiative and mechanical equilibrium, and the opacity is due to absorption from hydrogen, helium, and $\mathrm{H}^{-}$and from electron scattering. No account is taken of line blanketing. An estimate is made of the maximum changes that might occur as a result of line blanketing, and of where each spectral type should be written on the theoretical curve which relates bolometric corrections, $B C$, to effective temperature, $T_{e}$. For the purpose of estimating stellar radii from observed visual absolute magnitudes, it is necessary to know the quantity $B C+10 \log T_{e}$ as a function of spectral type. It is shown that this quantity is sensitive neither to the details of the model construction nor to spectral type. Consequently, the present theoretical results are adequate for finding radii. It is shown that the present estimates of absolute visual magnitude, $M_{v}$, and mass for B-type stars are consistent with the estimates of $T_{e}, B C$, and $\log g$ (the acceleration of gravity) that may be made from model atmosphere studies.

The problem of identifying a model atmosphere with a real star is considered and it is shown that the strengths of some of the spectral lines which are used for the classification of early B-type and O-type stars are sensitive to such parameters as the extent of the atmosphere and turbulence. Thus at types about B1 and earlier it is not possible to define a unique relationship between spectral type and effective temperature. The net radiation field is not necessarily the dominant factor controlling the strengths of the lines which are used for classification purposes.

\section{The HR Diagram}

The HR diagram is formed by entering stars in a diagram according to the coordinates spectral type and visual absolute magnitude. The spectral type is assigned from an evaluation of the detail that is displayed in the spectrum. The visual absolute magnitude may be assigned from the strengths of some spectral features which have been calibrated in terms of visual absolute magnitude, or it may be assigned from other information such as the apparent magnitude of the star, corrected for interstellar absorption, and the knowledge that the star is a member of a group which is at a known distance. Because the stars do not scatter all over the HR diagram but tend to lie on rather well-defined sequences, it appears that the empirically selected parameters may be related in a unique manner to the fundamental quantities 
which characterize the star. For a star of given mass the two basic parameters are thought to be the rate of radiating energy per unit surface area and the radius of the star. The rate of radiating energy may be represented by a number called the effective temperature and the radius by the acceleration of gravity at the star's surface. The bolometric absolute magnitude gives a combined measure of both parameters, effective temperature and radius.

In order to relate the observed visual absolute magnitude and spectral type to the theoretical parameters, radius and effective temperature, it is necessary to know the relationship between spectral type and effective temperature and that between bolometric correction and effective temperature at each point in the HR diagram.

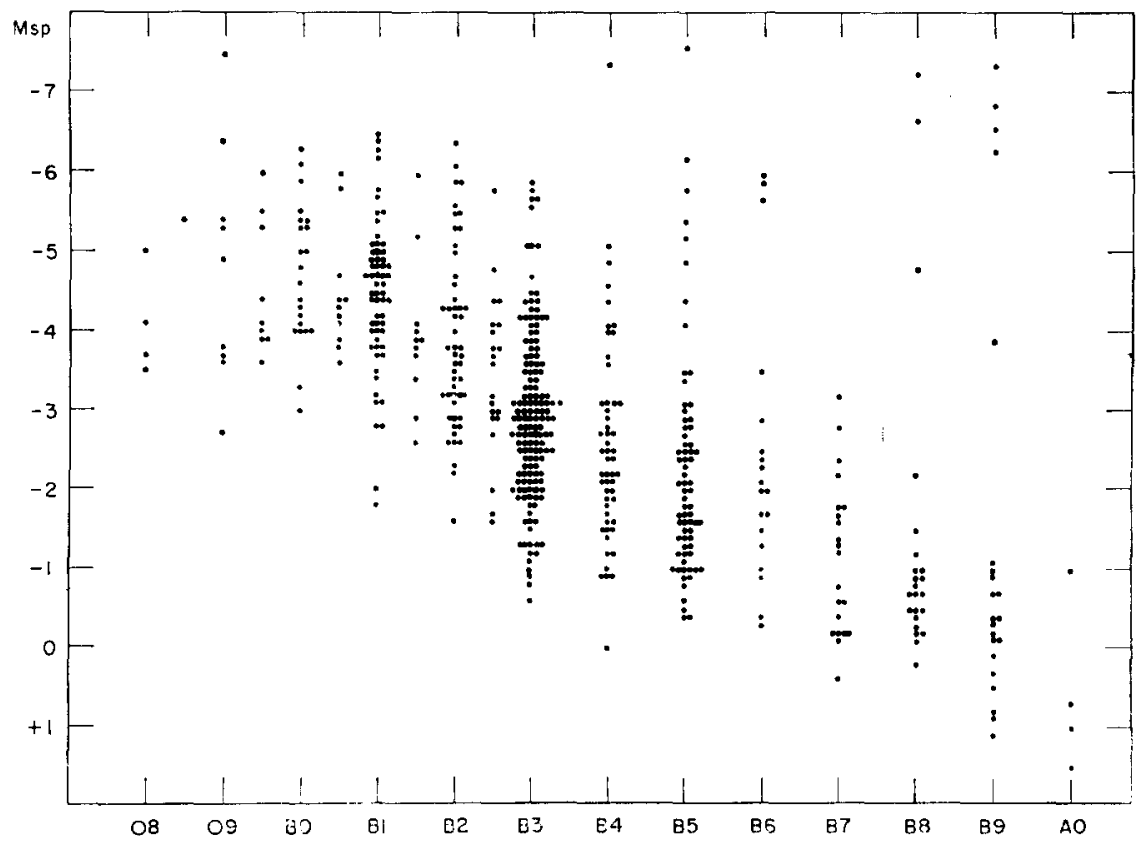

FIG. 1. The HR diagram for B-type stars in the neighborhood of the Sun according to PETRIE and LEE (1965).

Since the $\mathrm{O}$ - and B-type stars radiate most of their energy in a wavelength region which is inaccessible to observation from the surface of the Earth, owing to absorption by ozone and other gases in the Earth's atmosphere, it is necessary to use theoretical considerations to estimate the desired relationships. Our observed energy distribution curves for the $\mathrm{O}$ and $\mathrm{B}$ stars do not give an adequate base from which to extrapolate to the total flux. The purpose of this paper is to look at the question of how we may map an observed $H R$ diagram for $B$ stars onto a theoretical diagram of bolometric magnitude, or radius, vs. effective temperature.

In order to orientate ourselves, an observed HR diagram for B stars in the neighbourhood of the Sun is shown in Fig. 1. This diagram has been prepared by Petrie and Lee and it is based on the most recent spectral types and absolute magnitudes determined at the Dominion Astrophysical Observatory. A very striking characteristic is the wide scatter in absolute magnitude that exists for the early B-type stars. It seems unlikely that this spread is due entirely to the inherent 
difficulties of determining accurate visual absolute magnitudes and spectral types for the B stars. An insight into the meaning of this spread would be useful. CrawrorD (1958) has suggested that part of the spread might be due to the different ages of the stars.

From the definition of bolometric absolute magnitude one may show that for a star with radius $R$ we have

$$
\log R / R_{\odot}=0 \cdot 20\left[42 \cdot 24-\left(B C+\log T_{e}\right)-M_{v}\right],
$$

where $R_{\odot}$ is the solar radius. The constant in equation (1) results from adopting +4.62 as the bolometric absolute magnitude of the Sun and taking the effective temperature of the Sun to be $5785^{\circ} \mathrm{K}$. On the other hand, from the definition of surface gravity

$$
\log R / R_{\odot}=0.5\left[\log \left(\boldsymbol{m} / \boldsymbol{m}_{\odot}\right)-\log \left(g / g_{\circ}\right)\right],
$$

where $\boldsymbol{m}$ is the mass of the star, $\boldsymbol{m}_{\odot}$ that of the Sun, while $g$ and $g_{\odot}$ are the accelertions of gravity of the star and the Sun, respectively.

The quantities $\left(B C+10 \log T_{e}\right)$ and $\log \left(g / g_{0}\right)$ may be estimated for B-type stars from model atmosphere computations. If the model atmosphere gives a consistent representation of a B-type stellar atmosphere, equations (1) and (2) should give the same answer when they are used with well-determined visual absolute magnitudes and masses. We shall see what in fact is the case, and try to estimate the reliability of our theoretical parameters $\left(B C+10 \log T_{e}\right)$ and $g$.

\section{Bolometric Corrections and Effective Temperatures from B-type Model Atmospheres}

The effective temperature is defined by the following equation

$$
\sigma T_{e}=\pi \int_{0}^{\infty} F_{v}(\tau) \mathrm{d} v
$$

Here $F_{v}(\tau)$ is the net monochromatic flux at frequency $v$ at depth $\tau$ in a model atmosphere and $\sigma$ is the radiation constant. If the model atmosphere is in radiative equilibrium, the value of the integral is independent of $\tau$. The temperature law in each of the models under discussion has been adjusted so that the integrated flux is constant to within 0.5 per cent throughout the parts of the model that are important for producing the emergent spectrum $F_{v}(0)$. The models are thus in radiative equilibrium. The pressure structure of the models has been found by postulating that the models be in mechanical equilibrium, account being taken of the radiation pressure gradient. The ionization and excitation equilibria are computed assuming local thermodynamic equilibrium.

From the definition of bolometric correction and upon the assumption that the bolometric correction of the Sun is -0.07 magnitude the bolometric correction for any other model or star is

$$
B C=2.5 \log \frac{\int F_{\lambda}(*) p_{\lambda} \mathrm{d} \lambda}{\int F_{\lambda}(\odot) p_{\lambda} \mathrm{d} \lambda}-10 \log \frac{T_{e}\left(^{*}\right)}{T_{e}(\odot)}-0.07 .
$$

In equation (4) $p_{\lambda}$ is a normalized photovisual sensitivity function. For the present calculations the photovisual sensitivity function given by ALLEN (1955) and the monochromatic flux from the Sun listed by UssöLd (1955) are adopted. It 
44 Suggestions Regarding the Effective Temperatures and Bolometric Corrections for B-type Stars

follows that for model atmospheres the bolometric correction may be computed from the following equation:

$$
B C=2 \cdot 5 \log \left[\int F_{\lambda}(\operatorname{model}) p_{\lambda} \mathrm{d} \lambda\right]-10 \log T_{e}+20 \cdot 72 .
$$

Here $F_{\lambda}$ is the emergent flux in ergs $\mathrm{cm}^{-2} \mathrm{sec}^{-1}$ per angstrom interval. The quantity $F_{\lambda}$ may be found from most model atmosphere computations.

Equations (3) and (5) were used to compute the effective temperatures and bolometric corrections for fourteen model atmospheres which are believed to represent B-type stars. In each model the mass fraction of hydrogen, $X$, is 0.68 , that of helium, $Y$, is $\mathbf{0} \cdot 32$. The only sources of opacity in the models are continuous absorption from hydrogen, neutral and ionized helium, and $\mathrm{H}$ - and electron scattering. The models (UNDERHILL, 1962a, 1963 and unpublished) are in radiative and mechanical equilibrium and the radiation pressure gradient is taekn into account. The theoretical continuous spectrum is calculated taking into account the effect

TABLE 1

Models without Line Blanketing and with $X=0.68, Y=0.32$

\begin{tabular}{|c|c|c|c|c|c|c|c|c|c|c|}
\hline \multirow{2}{*}{$\begin{array}{c}T \text { at } \\
\tau \sim 0.1 \\
\text { degrees }\end{array}$} & \multicolumn{5}{|c|}{$g=10,000 \mathrm{~cm} \mathrm{sec}-2$} & \multicolumn{5}{|c|}{$g=5000 \mathrm{~cm} \mathrm{sec}-2$} \\
\hline & $\begin{array}{c}\text { Model } \\
\text { no. }\end{array}$ & $\begin{array}{c}T_{e} \\
\text { degrees }\end{array}$ & $D$ & $\begin{array}{c}\text { Spectral } \\
\text { type }\end{array}$ & $\begin{array}{c}B C \\
\text { mag. }\end{array}$ & $\begin{array}{c}\text { Model } \\
\text { no. }\end{array}$ & $\begin{array}{c}T_{e} \\
\text { degrees }\end{array}$ & $D$ & $\begin{array}{c}\text { Spectral } \\
\text { type }\end{array}$ & $\begin{array}{c}B C \\
\text { mag. }\end{array}$ \\
\hline 10,000 & 73 & 12,690 & $0 \cdot 452$ & B9 & -0.92 & & & & & \\
\hline 12,000 & 66 & 15,333 & 0.324 & B6 & $-1 \cdot 37$ & 119 & 14,870 & $0 \cdot 344$ & B7 & $-1 \cdot 28$ \\
\hline 15,000 & 55 & 19,215 & 0.210 & B3 & -1.91 & & & & & \\
\hline 20,000 & 63 & 25,673 & 0.126 & $\mathrm{~B} 2$ & $-2 \cdot 59$ & 148 & 25.894 & $0 \cdot 122$ & B2 & $-2 \cdot 61$ \\
\hline 22,000 & 89 & 27,820 & $0 \cdot 108$ & $\mathbf{B l} \cdot \mathbf{5}$ & $-2 \cdot 78$ & 143 & 27,777 & 0.093 & Bl & $-2 \cdot 74$ \\
\hline 24,000 & 97 & 30,027 & 0.075 & B0.5 & -2.93 & 146 & 29,194 & $0 \cdot 070$ & B0 & $-2 \cdot 81$ \\
\hline 25,200 & 64 & 31,023 & 0.068 & Bo & $-2 \cdot 96$ & 145 & 30,355 & $0 \cdot 056$ & 09.5 & $-2 \cdot 87$ \\
\hline 30,000 & 98 & 33,947 & 0.037 & O9 & $-3 \cdot 14$ & 140 & 33,411 & 0.024 & 08 & $-3 \cdot 07$ \\
\hline
\end{tabular}

of electron scattering on the source function. No line blanketing is included. The resulting effective temperatures and bolometric corrections are given in Table 1 .

An estimate of the equivalent spectral type of each model may be obtained from the computed Balmer jump, $D$, using the observed relationship between $D$ and spectral type found by Chalonge and Divan (1952). The first column of Table 1 lists the temperature at a representative layer in the atmosphere. This temperature characterizes the level of excitation in the atmosphere.

The relationship between $D$ and spectral type for B-type stars of luminosity classes III, IV, and V is shown in Fig. 2. Here stars from Table 2 of the paper by Chalonge and Divan have been plotted and a smooth curve has been drawn through the points. This curve is used to assign the equivalent spectral type of each model.

The theoretical relationship between $B C$ and $T_{e}$ for the models is shown in Fig. 3 . Here the models with $g=10^{4}$ are plotted as solid points, those with $g=5000$ as open circles. It is clear that a single curve is defined. The greatest uncertainty comes in assigning a spectral type to each part of the curve. The spectral types for the models with $g=10^{4}$ have been placed in the diagram. The relation compiled by HARRIS (1963) between bolometric correction and effective temperature for the early-type stars is shown as a broken line. 


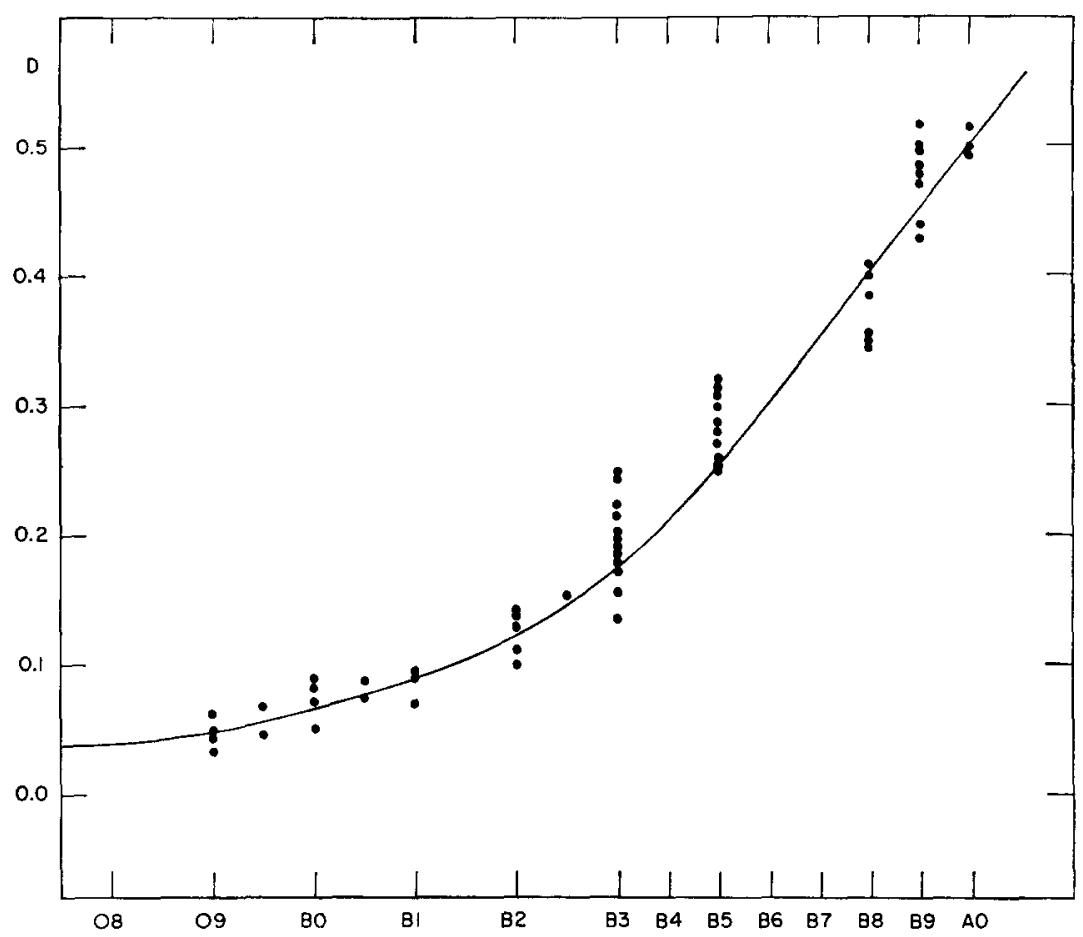

Fig. 2. The observed relationship between $D$ and spectral type according to Chalonge and Divan.

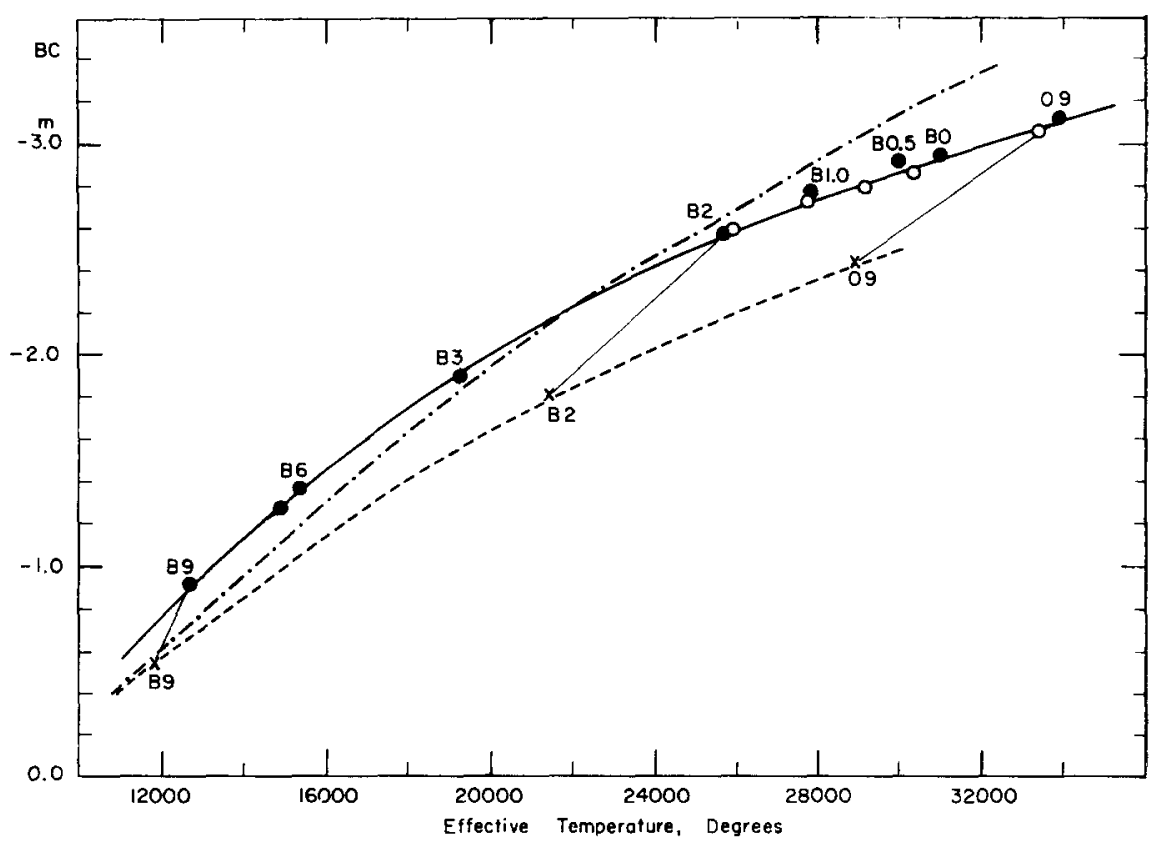

Fra. 3. The theoretical relationship between $B C$ and $T_{e}$. The models with $g=10^{4}$ are plotted as solid points, those with $g=5000$ as circles. The estimated changes caused by line blanketing are shown by crosses. The relationship compiled by Harris is shown as a broken line. 
The present models have been constructed with no consideration of the opacity due to line blanketing. It is, however, certain that line blanketing will greatly reduce the emergent flux in the ultraviolet wavelengths, where the models are rather transparent, from that presently predicted. In order to estimate the changes in the bolometric correction and effective temperature that might result, the effective temperature was recomputed using a truncated flux integral, the emergent flux between $\lambda$ 911.6 (the longward side of the Lyman limit) and $\lambda 1268$ being put equal to zero. The three points entered as crosses resulted. The revised effective temperatures and bolometric corrections and their differences from the data of Table 1 are given in Table 2.

We shall presume that the bolometric corrections and effective temperatures for revised models in which line blanketing has been taken into account in a more correct manner will lie along the lines joining the crosses to the points for the same models. A numerical experiment to investigate line blanketing in a more satisfactory manner is now being worked out. We should note that the temperatures and pressures in the outer parts of the present models do predict $\mathrm{H} \gamma$ lines very like those which are

TABLE 2

Estimates of the Effects of Line Blanketing

\begin{tabular}{c|c|c|c|c}
\hline $\begin{array}{c}\text { Model } \\
\text { no. }\end{array}$ & $\begin{array}{c}\text { Revised } \\
T_{e}\end{array}$ & $\begin{array}{c}\text { Revised } \\
B C\end{array}$ & $\Delta T_{e}$ & $\triangle B C$ \\
\hline & degrees & mag. & degrees & mag. \\
73 & 11759 & -0.54 & -931 & $+0 \cdot 38$ \\
63 & 21371 & -1.80 & -4302 & $+0 \cdot 79$ \\
98 & 28916 & -2.44 & -5031 & +0.61 \\
\hline
\end{tabular}

observed in sharp-lined main-sequence stars which have types the same as the assigned spectral types of the models. Consequently, the temperature and pressure structure must not be changed drastically when line blanketing is incorporated. Otherwise the agreement between observation and computation will disappear.

The estimated changes in the bolometric correction and the effective temperature which may be caused by line blanketing look rather large. However, the change in the quantity $\left(B C+10 \log T_{e}\right)$, which appears in equation (1), is not large. This may be seen from the values which are listed in Table 3 . Values found from the data given by Harris are presented for spectral types B9, B2, and 09. The differences between all these numerical results are not sufficiently great to prevent the estimate of radius by means of equation (1).

It is recommended that the set of theoretical bolometric corrections and effective temperatures given in Table 1 be adopted. They have the advantage that they come from a homogeneous set of calculations. There is some uncertainty where the spectral types should be written on the curve which relates bolometric correction to effective temperature. However, the resulting uncertainty in $\left(B C+10 \log T_{e}\right)$ will almost certainly be less than the uncertainty in estimating $M_{v}$ for any given $B$-type star. The quantity $\left(B C+10 \log T_{e}\right)$ does not change rapidly with spectral type, nor is it particularly sensitive to the details of the model computations.

No serious error in $\log R / R_{\odot}$ would be introduced by using the bolometric corrections and effective temperatures compiled by Harris. However, these values do not 
form a homogeneous set. The selected bolometric corrections and effective temperatures have been obtained by different, arbitrary interpolations between values grounded on observations of stars of later spectral types and values suggested from models which were computed at an earlier date and which are not so satisfactory as the present models in regard to meeting the condition of radiative equilibrium. In particular, the computations indicate that the effective temperature of an early B-type star should be rather higher than the temperature which is representative of the level of excitation in the outer layers of the atmosphere. The "effective temperatures" suggested by Harris appear to be more like the characteristic temperatures given in the first column of Table 1, than true effective temperatures. From a logical point of view, the effective temperatures and bolometric corrections suggested by WILDEY (1963) are not more satisfactory.

TABLE 3

Some Values of The QuanTtTy $\left(B C+10 \log T_{e}\right)$

\begin{tabular}{|c|c|c|c|c|c|c|c|}
\hline \multirow[b]{2}{*}{$\begin{array}{l}\text { Model } \\
\text { no. }\end{array}$} & \multirow[b]{2}{*}{$\begin{array}{c}\text { Spectral } \\
\text { type }\end{array}$} & \multirow{2}{*}{\multicolumn{2}{|c|}{$\begin{array}{cc}B C+10 \log T_{e} \\
\text { no line } & \text { estimated } \\
\text { blanketing } & \text { blanketing }\end{array}$}} & \multirow[b]{2}{*}{$\begin{array}{c}\text { Spectral } \\
\text { type }\end{array}$} & \multicolumn{3}{|c|}{ HARRIS (1963) } \\
\hline & & & & & $\begin{array}{c}T_{e} \\
\text { degrees }\end{array}$ & $\begin{array}{c}B C \\
\text { mag. }\end{array}$ & $B C+10 \log T_{e}$ \\
\hline 73 & B9 & $40 \cdot 11$ & $40 \cdot 06$ & B9 & 12,400 & -0.66 & $40 \cdot 27$ \\
\hline 63 & B2 & $41 \cdot 50$ & $41 \cdot 50$ & $\mathrm{~B}^{2}$ & 22,100 & $-2 \cdot 23$ & $41 \cdot 21$ \\
\hline 98 & 09 & $42 \cdot 17$ & $42 \cdot 17$ & O9 & 31,900 & $-3 \cdot 34$ & $41 \cdot 70$ \\
\hline
\end{tabular}

\section{The Consistency of the Recommended Bolometric Corrections and Effective Temperatures}

Approximate spectral types have been assigned to the models from the computed change in intensity at the Balmer limit. By comparing the computed $\mathrm{H}_{\gamma}$ profiles (UNDERHILL, 1962b and unpublished) with observed profiles, it may be verified that the models produce $\mathrm{H} \gamma$ profiles closely resembling those in mainsequence B-type stars of the same type. There is some evidence that the cores which are observed for the hydrogen lines in sharp-lined $B$ stars cannot be reproduced unless the outer atmospheres of the models are treated in more detail than in the present models. The necessary changes to the models are in the direction of adding a comparatively low temperature, low pressure halo in which microturbulence occurs, but these changes do not change the value of the effective temperature or the bolometric correction by a significant amount. So far as the wings of the hydrogen lines are concerned, the present models appear to represent B-type stars of luminosity classes IV and V well.

Minalas (1963) has also computed a series of models for B-type stars. Some of his models closely resemble the present models. $\mathrm{His} \mathrm{H} \gamma$ profiles are very similar to the theoretical $\mathbf{H} \gamma$ profiles referred to here. Mihalas' models with $\log g=4 \cdot 5$ give $\mathrm{H}_{\gamma}$ profiles which are broader than the observed $\mathrm{H}_{\gamma}$ profiles in 10 Lacertae, $09 \mathrm{~V}$, $\gamma$ Pegasi, B2IV, or $\iota$ Herculis, B3V. One may conclude that in B stars of luminosity classes IV and $V$ the effective $g$ is probably not greater than $3.2 \times 10^{4} \mathrm{~cm} \mathrm{sec}$ s. $^{-2}$. In fact the best fit in the wings of $\mathrm{H}_{\gamma}$ between observation and theory is obtained with the models in which $g=10^{4} \mathrm{~cm} \mathrm{sec}-2$ or a little less. 
It may be concluded from this work that the acceleration of gravity in the atmospheres of B-type stars of luminosity class $V$ is near $10^{4} \mathrm{~cm} \mathrm{sec-2}$ and that in luminosity class IV stars it is about $5 \times 10^{3} \mathrm{~cm} \mathrm{sec}^{-2}$. It seems pretty certain that the acceleration of gravity is not so great as $3.2 \times 10^{4} \mathrm{~cm} \mathrm{sec}^{-2}$ in the atmospheres of main-sequence stars of type B. These estimates of $g$ should represent the masses and radii of these stars. Only if microturbulent motions contribute a term to the equation of mechanical equilibrium that is comparable to $g /(\kappa+\sigma)$, where $\kappa+\sigma$ is the mean opacity coefficient per gram of star material, will it be necessary to consider these estimates to be a lower limit to the true acceleration of gravity in the stellar atmospheres. The radiation pressure term has been taken into account in making these estimates of $g$.

JoHNSON and IRIARTE (1958) have proposed visual absolute magnitudes for the $B$ stars. It is possible that these absolute magnitudes are somewhat too bright for the BI and earlier stars, but we shall adopt these values for the purpose of

TABLF 4

Estimates of THE Radil of B-TYpe StaRs

\begin{tabular}{|c|c|c|c|c|c|c|}
\hline \multirow{2}{*}{$\begin{array}{c}\text { Spectral } \\
\text { type }\end{array}$} & \multirow{2}{*}{$\begin{array}{c}M_{v} \\
\text { mag. }\end{array}$} & \multirow{2}{*}{$\begin{array}{c}\text { Mass } \\
m_{\odot}\end{array}$} & \multirow{2}{*}{$\begin{array}{c}\log R / R_{\odot} \\
\text { eq. (1) }\end{array}$} & \multicolumn{3}{|c|}{$\log R / R_{\odot}$, eq. (2) } \\
\hline & & & & $g=5 \times 10^{3}$ & $g=10^{4}$ & $g=2 \times 10^{4}$ \\
\hline B5 V & $-1 \cdot 0$ & 4.7 & 0.55 & 0.71 & 0.56 & $0 \cdot 35$ \\
\hline B5 IV & $-1 \cdot 8$ & & $0 \cdot 71$ & & & \\
\hline $\mathrm{B} 3 \mathrm{~V}$ & $-1 \cdot 7$ & $10 \cdot 2$ & $0 \cdot 60$ & $0 \cdot 87$ & 0.72 & 0.57 \\
\hline B3 IV & $-2 \cdot 5$ & & 0.76 & & & \\
\hline $\mathrm{Bl} \mathrm{V}$ & $-3 \cdot 6$ & $13 \cdot 6$ & 0.80 & 0.94 & 0.79 & $0 \cdot 64$ \\
\hline BI IV & $-4 \cdot 1$ & & $0 \cdot 90$ & & & \\
\hline O9 V & $-4 \cdot 6$ & $23 \cdot 3$ & 0.93 & $1 \cdot 05$ & $0 \cdot 90$ & $0 \cdot 75$ \\
\hline O9 IV & $-5 \cdot 4$ & & $1 \cdot 09$ & & & \\
\hline
\end{tabular}

estimating the radii of $\mathrm{B}$ stars, and we shall use the effective temperatures and bolometric corrections recommended here. Pearce (1957) has determined the average masses of B-type stars from 30 well studied eclipsing binaries. From his compilation we shall adopt masses for main sequence stars of types B5, B3, B1, and 09 for the purpose of estimating radii from our spectroscopically determined values of $g$. The values of $\log R / R_{\odot}$ resulting from equations (1) and (2) are given in Table 4.

At types B5, B1 and 09 the adopted masses and visual absolute magnitudes together with the data suggested from model atmosphere studies predict consistent values of radius. A reduction of the absolute magnitudes of the 09 stars by 0.5 mag. would make the agreement at this spectral type even better. The two estimates of radius do not coincide so well in the case of the B3 stars. Here better agreement might be obtained if larger values of $g$ were adopted, or if the adopted mass was reduced to about 8 solar masses. In the case of $\mathrm{B} 3$ stars there is little reason to suggest revising the visual absolute magnitudes.

This test of the consistency of our information about masses, visual absolute magnitudes, and radii for B-type stars is not very stringent. However, it is useful to realize that no serious clashes exist among the proposed values. The present spectroscopic estimates of $g$ make use of improved theories of the Stark broadening of hydrogen lines by protons and by electrons. Thereby the spectroscopic values of 
$g$ are reduced from the first estimates that were made in this way, and the deduced radii are in reasonable agreement with the radii deduced from other data.

\section{The Spectral Classification Problem Posed by the Models}

It is generally considered that a spectral type represents the average values of temperature and pressure in the stellar atmosphere, and that a quantitative relationship may be set up between the basic parameters effective temperature and acceleration of gravity by identifying models with real stars. The models have been identified with B-type stars by means of the predicted discontinuity at the Balmer limit and by the strength and shape of $\mathrm{H} \gamma$. That is, on the basis of identity between a small part of the predicted spectral distribution due to hydrogen and certain observed spectral details. However, a spectral type is not assigned on the basis of the hydrogen spectrum alone. In fact, in the early B-type stars, in particular, it depends rather critically on the apparent relative strengths of lines from $\mathrm{He}$, $\mathrm{C} \mathrm{II}, \mathrm{N}$ II, O II, Si II, Si III, and Si IV. In the O-stars the relative strengths of certain He I and He II lines are an important criterion. If a model is to represent a B-type star it should reproduce accurately all the spectral details which characterize the assigned spectral type.

The present models do not reproduce well the observed secondary lines when the lines are strong, thus observable. In B-type stars one cannot observe easily "weak", lines, that is lines on the Doppler part of the curve of growth, because such lines are very shallow. A comparison between observed and computed equivalent widths of Si II $\lambda 4130$, Si III $\lambda$ 4552, and Si IV $\lambda 4088$ over the spectral range B9 to O9 shows that at the spectral types where the lines are found to be strong, thus conveniently measurable, the lines are far stronger than can be predicted using a reasonable estimate of the abundance of silicon and models which consist of plane parallel layers in mechanical equilibrium. The explanation appears to be that when the lines are strong they are broadened and strengthened by microturbulence (UNDERHILL, 1963; DE Groot and UNDERHILL, 1964). This conclusion was reached from a study of equivalent widths, and it has been confirmed by a study of line profiles in 10 Lacertae and in $\gamma$ Pegasi. Further confirmation would be desirable. It could be obtained by studying the profiles of lines in several sharp-lined B-stars. This is a demanding observational program which cannot be solved immediately.

The probable existence of microturbulence in the atmospheres of B-type stars poses the interesting question of the origin of the motions, seeing that one does not expect a classical hydrogen convection zone, nor a strong convection zone due to the first or second ionization of helium. Perhaps the turbulence results from the shear motion due to differential rotation. Whatever the origin of the turbulence, the recognition that the strengths of the secondary lines which are used for the spectral classification of B-type spectra are strongly dependent on a third parameter, the amount of motion in the atmosphere, makes a simple interpretation of spectral type in terms of two parameters, effective temperature and acceleration of gravity (or electron pressure), impossible.

The relationship which comes most into question is that between spectral type and effective temperature, particularly for the $\mathrm{O}$ - and $\mathrm{B}$-type stars where one cannot directly observe a sufficiently important part of the total radiation field from the star to lead to a reliable estimate of the effective temperature according to equation 
(3). By analyzing the spectrum, one may obtain an estimate of the excitation temperature and of the ionization temperature in the part of the atmosphere which produces the observed spectral lines, but there is no direct way of relating this temperature to the effective temperature which is a measure of the total radiative flux crossing each unit surface area. In order to relate the spectroscopically estimated excitation or ionization temperature to the effective temperature one must be able to tie the physical conditions in the part of the atmosphere where the lines are formed to those in the part of the atmosphere where radiative equilibrium, local thermodynamic equilibrium, and mechanical equilibrium are adequate constraints on the equation of state. One necessary step would be to specify how the state of motion in the line-forming layers of the atmosphere affects the level of excitation and ionization. Also one must consider dilution effects in certain spectra.

These problems would not be important in the study of the HR diagram if we were sure that in all spectral types our criteria for spectral classification were sensitive dominantly to the constraints radiative equilibrium, local thermodynamic equilibrium, and mechanical equilibrium and to no other constraining factors. Unfortunately some of the criteria used for spectral classification in the early B-type stars are sensitive to dilution effects and to turbulence. One striking example is the use of the relative strengths of $\mathrm{He} \mathrm{I}$ and $\mathrm{He}$ II lines as a classification criterion for the $\mathrm{O}$-stars. It may readily be seen that the He I lines are subject to dilution effects, for the lines from the $2^{3} \mathrm{~S}$ and $2^{3} \mathrm{P}$ levels strengthen at the expense of the lines from other levels. The He I lines are formed in a shell or extended atmosphere around the star. Very likely their strength is also strongly influenced by the amount of turbulence present in this shell. On the other hand, the He II lines which are used for classification come from the $n=4$ level of $\mathrm{He}^{+}$which has an excitation potential of $51 \cdot 0 \mathrm{~V}$. These He II lines come from deep in the stellar atmosphere. The relative strength of the two sets of lines is certainly a well-defined parameter, but it cannot be related uniquely to effective temperature because the strengths of the He I lines depend strongly on parameters which are not necessarily closely related to effective temperature.

It seems very probable that all $\mathrm{O}$-stars have extended atmospheres or shells of some amount. The star 10 Lacertae is well known as a sharp-lined $09 \mathrm{~V}$ standard star. However, when the observed absolute strengths of lines from levels of high excitation potential such as the He II and O III lines are compared with the predicted strengths from a model, which appears from the low excitation (in this context) spectrum of hydrogen to represent an 09 star, they are found to be too strong. According to the He II and O III lines, 10 Lacertae is perhaps an 08 or an 07 star. The type 09 has been assigned mostly on account of the sharp lines of O II, Ne II, Si III, Si IV, etc., that are seen. These lines, and the sharp hydrogen cores, come from a shell.

The system of spectral classification for the early-type stars remains well-defined because it is based on empirically selected features that can be arranged in a unique order. What has become lost is the possibility of relating uniquely the quantity we call spectral type to effective temperature, for the spectral type now is a measure of something more than the quality of the radiation field passing through the atmosphere and the electron pressure in the atmosphere. These difficulties begin at about spectral type B3. It is about here that dilution effects like the well-known effects in the He I spectrum begin to affect the classification system. Other spectra, 
notably Si III, the $\lambda 4552$ multiplet, show dilution effects like those in the He I spectrum. Furthermore, some lines are strengthened with respect to others by the presence of microturbulence even in the case of the so-called sharp-lined B-stars. At present insufficient theoretical spectra and too few observed profiles and equivalent widths are at hand to elucidate this problem completely. However, it seems quite probable that some of the rather great width of the main-sequence for early B-type stars (see for instance Petrie and Moyls, 1956, or Crawford, 1958) arises because the same spectral type or broadband intrinsic color does not isolate stars of a given effective temperature, but rather only those having a certain combination of level of excitation and extended atmosphere. It is reasonable that these stars should have different absolute magnitudes.

The problem posed by early B- and O-type spectra is to find classification criteria which permit a unique relationship to be established between spectral type and effective temperature. The model atmosphere computations indicate that the shape and the equivalent width of $\mathrm{H} \gamma$ will do. However, these are not useful criteria in practice because one cannot determine from observations the profile $\mathrm{H} \gamma$ would have had if the star was single and standing still instead of rotating rapidly and very likely being a member of a binary system. Unfortunately, few if any, early type stars appear to be like the relatively simple models with which we try to relate observed phenomena to the basic parameters by which we characterize stars.

The first attempts at comparing details in the spectra of normal sharp-lined Bstars with the detailed theoretical stellar spectra computed from models which appear to give a good representation of main-sequence stars so far as the hydrogen spectrum is concerned have demonstrated that motion in the atmosphere and the extent of the atmosphere have an important effect on the resulting spectrum. One wonders indeed what a normal star is like and whether some of our difficulties about strong-lined and weak-lined stars at later types are not further evidence that the "normal" star is not one with an atmosphere only in radiative, thermodynamic and mechanical equilibrium, but one in which the coupling between the mechanical motion and the level of excitation in the atmosphere also plays an important part.

\begin{tabular}{|c|c|c|c|c|}
\hline \multicolumn{5}{|c|}{ References } \\
\hline AlLen, C. W. & . & . & 1955 & Astrophys. Quantities (London: Athlone Press), 178. \\
\hline Chalonge, D., and I & Divan, $\mathbf{L}$. & . & 1952 & Ann.d'Ap., 15, 201 \\
\hline Crawford, D. L. & . & . & 1958 & $A p . J ., 128,185$ \\
\hline DE Groot, M., and I & UNDERHILL, & A. $\mathbf{B}$. & 1964 & B.A.N., 17, 280. \\
\hline Harits, D. L. . & • & - & 1963 & $\begin{array}{l}\text { Basic Astronomical Data, ed: K. Aa. Strand } \\
\text { (Chicago: University of Chicago Press), p. } 267 .\end{array}$ \\
\hline JoHNSON, H. L., and & d IrIarte, B & & 1948 & Lowell Obs. Bull., No. 91. \\
\hline Mihalas, D. & . & . & 1963 & Thesis, California Institute of Technology. \\
\hline Pearce, J. A. . & . & . & 1957 & J.R.A.S. Canada, 51, 59; Contr. Dom. Ap. Obs., 53. \\
\hline Petrie, R. M., and & LEE, E. K. & . & 1965 & In preparation. \\
\hline Petrie, R. M., and & MoyLs, B. A & & 1956 & Pub. Dom. Ap. Obs., 10, 287. \\
\hline UNDERHILL, A. B. & . & . & $1962 \mathrm{a}$ & Pub. Dom. Ap. Obs., 11, 433. \\
\hline UNDERHILL, A. B. & . & . & $1962 \mathrm{~b}$ & Pub. Dom. Ap. Obs., 11, 467. \\
\hline UNDERHILL, A. B. & . & - & 1963 & B.A.N.17, 161 . \\
\hline UNSÖLD, A. & . & . & 1955 & $\begin{array}{l}\text { Physik der Sternatmosphären (Berlin: Springer } \\
\text { Verlag), p. } 37 .\end{array}$ \\
\hline WILDEY, R. L. . & . & - & 1963 & Nature, 199, 988. \\
\hline
\end{tabular}

